\title{
Dysfunctional immunity and microbial adhesion molecules in smoking-induced pneumonia
}

Mathew Suji Eapen ${ }^{1}$, Pawan Sharma ${ }^{2,3}$, Yuben P. Moodley ${ }^{4,5,6}$, Philip M. Hansbro ${ }^{7,8,9}$, Sukhwinder Singh Sohal ${ }^{1}$

${ }^{1}$ Respiratory Translational Research Group, Department of Laboratory Medicine, College of Health and Medicine, University of Tasmania, Launceston, Tasmania, Australia, 7248

${ }^{2}$ Discipline of Medical Sciences, School of Life Sciences, University of Technology Sydney, Sydney, NSW, Australia, 2007,

${ }^{3}$ Woolcock Emphysema Centre, Woolcock Institute of Medical Research, University of Sydney, Sydney, NSW, Australia, 2037.

${ }^{4}$ Stem Cell Unit, Institute of Respiratory Health, Perth, Australia.

${ }^{5}$ Centre for Respiratory Health, School of Medicine and Pharmacology, University of Western Australia, Perth, Australia

${ }^{6}$ Dept of Respiratory Medicine, Fiona Stanley Hospital, Murdoch, Australia.

${ }^{7}$ School of Biomedical Sciences and Pharmacy, The University of Newcastle, Callaghan, Australia.

${ }^{8}$ Priority Research Centre for Healthy Lungs, Hunter Medical Research Institute, Lot 1 Kookaburra Circuit, New Lambton Heights, Newcastle

${ }^{9}$ Centenary Institute and University of Technology Sydney, Australia.

\section{Corresponding Author}

Dr Sukhwinder Singh Sohal

Respiratory Translational Research Group

Department of Laboratory Medicine, School of Health Sciences,

College of Health and Medicine, University of Tasmania

Locked Bag - 1322, Newnham Drive

Launceston, Tasmania 7248, Australia

Telephone number: +61363245434

Email: sssohal@utas.edu.au

Funding support: SSS is supported by Clifford Craig Foundation Launceston, Thoracic Society of Australia \& New Zealand (TSANZ), Boehringer Ingelheim COPD Research Award, PMH is supported by an NHMRC Principal Research Fellowship and a Brawn Fellowship, Faculty of Health, University of Newcastle. PS is supported by Rebecca L. Cooper Medical Research Foundation, Australia and Chancellors Fellowship Programme, University of Technology Sydney (UTS). 


\section{Dear Editor}

We read with interest the recent article by Larson-Casey et al., and the corresponding editorial by Quinton et al., published in the AJRCCM, wherein the authors demonstrate the protective role of macrophage Rac2 in attenuating cigarette smoke-induced pneumonia (1). We consider the mechanistic observations in the study are important considering that pneumonia plays a crucial role in inducing increased exacerbations in patients with COPD. We would like to take this further and suggest a wider discussion on new insights from airway wall cellularity, macrophage (M1/M2) phenotypic change and the role of epithelial adhesion molecules such as platelet activated adhesion factor (PAFR) and intercellular adhesion molecule 1 (ICAM-1) in this setting.

Interestingly, this dysfunctionality of the immune system is evident from our previous reports wherein a hypo-cellular airway wall was observed in smokers and COPD patients and further, either no change or rather a decrease in the key immune cells such as neutrophils, CD68+ macrophages, and CD4+ T cells were observed when compared to non-smoker controls (2). The only cells that showed some significant increase were CD8+ T cells, which have also recently been demonstrated to be dysfunctional in clearing both bacterial and viral infections in COPD patients. Neutrophils seem to be in the same setting, whose ineffectiveness in combating bacterial infections have now been reasoned by Larson-Casey et al.

We further defined macrophage populations and our findings suggests the existence of differential macrophage switching that occurs in airway wall compared to those in the lumen and alveolar macrophages (AMs) (3). In non-smokers airway wall macrophages are predominantly M2 (CD163+), which switch to a more M1 phenotype (CD68+ iNOS+ dual positive) in smokers and COPDs. In contrast, AMs switched towards a more M2 phenotype and was mainly driven by cytokines/chemokines that skewed towards a M2/Th2 profile (3). Furthermore, AMs from COPD patients had comparatively reduced iNOS expression compared to non-smokers, confirming dysfunctionality; a key reason for their inability to mount an effective response to infection and efferocytosis. We also found increases in Arginase-1 expression in bronchoalveolar lavage (BAL) macrophages in COPD, which is indicative of a shift towards an M2 phenotype (3). This is the first comprehensive human study so far in smokers and COPD (3). With respect to these, we are curious if Larson-Casey et al., have any information from their current study regarding M1/M2 macrophage populations in the context of Rac 2 activity and effects on the clearance of pneumococci? 
The other important mechanistic approach to microbial pathogenesis is the prerequisite step of adherence of pathogens to the respiratory mucosa. The interaction between the epithelial and bacterial surface occurs through phosphorylcholine (ChoP) a molecular mimic of PAF present on the bacterial surface. PAFRs are expressed on the respiratory epithelium, which increases in response to cigarette smoke $(4,5)$. Both pneumococci and Haemophilus influenzae can attach to epithelial PAFR through ChoP and are protected partly due to dysfunctional immune response (4). We also observed that ICS have the potential to increase epithelial PAFR expression, suggesting a potential mechanism that underpins steroid dependent pneumonia (6). Similarly we observed an epithelial increase in the rhinovirus adhesion molecule, ICAM-1, suggesting involvement of more than one receptor that facilitates such mechanisms (4).

Taken together, these observations suggests that macrophages seem to be the key cell population with potential to combat bacterial and viral infections. However, it is vital to understand variations in airway wall cellularity, macrophage plasticity, and epithelial attachment sites for both viruses and bacteria, which potentially are also upregulated in response to ICS treatment. We believe there is an urgent need for both further in-depth human and animal studies of what are the fundamental issues with COPD.

\section{References}

1. Larson-Casey JL, Gu L, Jackson PL, Briles DE, Hale JY, Blalock JE, Wells JM, Deshane JS, Wang Y, Davis D, Antony VB, Massicano AVF, Lapi SE, Carter AB. Macrophage Rac2 Is Required to Reduce the Severity of Cigarette Smoke-induced Pneumonia. American journal of respiratory and critical care medicine 2018.

2. Eapen MS, McAlinden K, Tan D, Weston S, Ward C, Muller HK, Walters EH, Sohal SS. Profiling cellular and inflammatory changes in the airway wall of mild to moderate COPD. Respirology 2017.

3. Eapen MS, Hansbro PM, McAlinden K, Kim RY, Ward C, Hackett TL, Walters EH, Sohal SS. Abnormal M1/M2 macrophage phenotype profiles in the small airway wall and lumen in smokers and chronic obstructive pulmonary disease (COPD). Sci Rep 2017; 7: 13392.

4. Eapen MS, Sohal SS. Understanding novel mechanisms of microbial pathogenesis in chronic lung disease: implications for new therapeutic targets. Clin Sci (Lond) 2018; 132: 375379.

5. Grigg J, Walters H, Sohal SS, Wood-Baker R, Reid DW, Xu CB, Edvinsson L, Morissette MC, Stampfli MR, Kirwan M, Koh L, Suri R, Mushtaq N. Cigarette smoke and plateletactivating factor receptor dependent adhesion of Streptococcus pneumoniae to lower airway cells. Thorax 2012; 67: 908-913.

6. Sohal SS. Inhaled corticosteroids and increased microbial load in COPD: potential role of epithelial adhesion molecules. The European respiratory journal 2018; 51. 
